\section{Low versus High I-I3I Dose for Remnant Ablation in Differentiated Thyroid Cancer}

Editor - We would like to comment on an article published by Doi et al. ${ }^{1}$ The report was based on a review of studies examining the effectiveness of low and high administered doses of radioiodine for the ablation of thyroid remnant among patients with differentiated thyroid cancer. Their analysis was based on a previously published systematic review conducted by Hackshaw et $\mathrm{al}^{2}$ which concluded that there is currently insufficient evidence to reliably say whether a low dose is associated with a lower ablation success rate than a high dose. Doi et al, however, use the same studies to conclude that a low dose is worse, but there are fundamental problems with their analysis and interpretation.

First, they combine studies with very different designs into a single meta-analysis producing a relative risk which appears to be precise and highly statistically significant. The relative risk of having a successful ablation in the low dose group compared to the high dose group was $0.73,95 \%$ confidence interval (CI) $0.61-0.81$. The 22 studies, on which this particular result is based, are a mixture of cohort studies, retrospective reviews of patient medical records, and randomized trials. It is inappropriate to combine these studies when the observational studies are likely to be affected by inherent biases and confounding, but the randomized trials are not. Indeed, meta-analyses of observational studies need to be interpreted with great care because the effect of potential confounding in each study could be magnified when the studies are combined, thus producing a spuriously precise effect. ${ }^{3}$ Sixteen of the 22 studies $(73 \%)$ were observational, so their results will contribute most to the pooled result. The best evidence must come from randomized clinical trials.

Second, when their analysis was restricted to the 6 randomized trials (in which no comparison was based on more than 150 patients in total), the pooled relative risk was 0.68 (95\% CI $0.43-1.07)$, which was not statistically significant ( $P$-value of 0.093). The correct interpretation is that, while there is some evidence of a lower ablation rate when using a low dose, the effect could be due to chance because the CI includes the no-effect value and $P>0.05$ (i.e., there could be no true difference). However, no comment was made on this.

Third, the dose level that defines 'low' and 'high' differs greatly between these trials. For example, a low dose could be 30 or $50 \mathrm{mCi}$ (a difference of two-thirds), and a high dose could be 50 or $100 \mathrm{mCi}$ (a difference of two-fold). Because of this, it is best to not combine all the trials to produce a single relative risk.
Once these considerations have been taken into account, there is no analysis that allows firm conclusions to be made about the comparison of low and high dose radioiodine in treating differentiated thyroid cancer. While some clinicians prefer to administer a high dose at present, it is misleading to rule out the use of a low dose based on current evidence. There are clear potential advantages, such as less time spent in isolation in hospitals and lower risk of future second malignancies. ${ }^{2}$ What is needed is a large randomized trial, and there are two such trials in progress in the UK and France, each based on several hundred patients. ${ }^{4}$ These trials will determine with sufficient certainty whether a low dose should be avoided, or could be used instead of a high dose.

\section{References}

1. Doi SA, Woodhouse NJ, Thalib L, Onitilo A. Ablation of the thyroid remnant and I-131 dose in differentiated thyroid cancer: a meta-analysis revisited. Clin Med Res 2007; 5:87-90.

2. Hackshaw A, Harmer C, Mallick U, Haq M, Franklyn JA. ${ }^{131}$ I activity for remnant ablation in patients with differentiated thyroid cancer: a systematic review. J Clin Endocrinol Metab 2007;92:28-38.

3. Egger M, Schneider M, Davey Smith G. Spurious precision? Meta-analysis of observational studies. BMJ 1998;316: 140-144.

4. Mallick U, Harmer C, Hackshaw A. The HiLo trial: a multicentre randomised trial of high- versus low-dose radioiodine, with or without recombinant human thyroid stimulating hormone, for remnant ablation after surgery for differentiated thyroid cancer. Clin Oncol (R Coll Radiol) 2008;20:325-326.

Allan Hackshaw, MSc Deputy Director Cancer Research UK \& UCL Cancer Trials Centre University College London 90 Tottenham Court Road (5th floor) London WIT 4TJ United Kingdom Email:ah@ctc.ucl.ac.uk

Ujjal Mallick, MD

Clinical Oncologist Northern Cancer Centre Newcastle General Hospital Westgate Road Newcastle upon Tyne NE4 6BE United Kingdom 